\title{
Polynomial Algorithm for Node Deployment in Hybrid Wireless Sensor Networks
}

\author{
Lili Zhang ${ }^{\mathrm{a}}$, Jing Yuan ${ }^{\mathrm{b}}$, Yingchi Mao ${ }^{\mathrm{c}}$, Xiwei Zhang ${ }^{\mathrm{d}}$, Guihai Chen ${ }^{\mathrm{e}}$ \\ ${ }^{a, b, d, e}$ Department of Computer Science and Technology, Nanjing University, Nanjing, China \\ ${ }^{a, c, d}$ College of Computer and Information, Hohai University, Nanjing, China
}

\begin{abstract}
When detecting a target or monitoring a physical phenomenon in a region, the deployment problem is fundamental in these applications. Traditionally, stationary sensor networks are deployed to carry out the sensing operations. It is well known that the mobility of sensor nodes can improve the coverage and the probability of the detecting, so we deal with the problem of detecting a target using hybrid sensor networks which contain both stationary sensors and mobile sensors. In this paper, to begin with, we prove that the node deployment problem is NP-complete. Then, one polynomial algorithm for node deployment in hybrid wireless sensor networks is proposed, which aims at minimizing the number of all sensors to reduce the cost. The simulations verify the efficiency of our algorithm.
\end{abstract}

Index Terms: hybrid wireless sensor networks; node deployment; reaction delay.

(C) 2011 Published by MECS Publisher. Selection and/or peer review under responsibility of the Research Association of Modern Education and Computer Science

\section{Introduction}

Wireless Sensor Networks (WSNs) are applied more and more in surveillance systems for detecting a target or monitoring a physical phenomenon in a region. In these applications, the sensor deployment directly influences resource management, routing, and security etc.. Random and deterministic deployments are two general categories of deployment in WSNs, and most deterministic deployment is grid-based. Sensor deployment strategy depends on the applications. For example, if it is to be used for surveillance, the coverage can be considered as an important factor when deploying sensors. And discrimination must be considered in target location problems. What we consider, in this paper, is the number of the sensors for reducing the cost. References [1] and [2] presented source-bounded optimization frameworks for sensor resource management under the constraints of coverage. Reference [3] focused on theoretical analysis, and formulated the node deployment in terms of cost minimization under coverage constraints but no discussion of strategies of node deployment. On the other hand, reference [4] gave the regular optimal pattern of static sensor deployment based on polygon, but it is used for homogeneous wireless sensor networks.

* Corresponding author.

E-mail address: ${ }^{\text {alilzhang@hhu.edu.cn }}$ 
Several recent studies pay attention on the hybrid wireless sensor networks which contain both static sensors and mobile sensors. For example, reference [5,6] analyzed the impact of mobility on detection delay and area coverage; Bisnik et al. [7] focused on the performance of detecting stochastic events using mobile sensors; Chin et al. [5] built an energy model and based on it proposed an analytic method to evaluate the detection latency by a collaborative sensing approach using nodes uncoordinated mobility; Xing et al. [8] explored the use of mobile sensors to address the limitation of static sensors for target detection and developed an optimal sensor movement scheduling algorithm; and Gandhi et al. [9] showed that a sample with $O\left(\frac{1}{\varepsilon} \log \frac{1}{\varepsilon}\right)$ sensor nodes is sufficient to catch any event for $0<\varepsilon<1$, if the sensors are deployed randomly. However, there are more theoretical analyses in the previous works but fewer practical strategies of node deployment under certain constraints, such as the delay time, in hybrid wireless sensor networks.

Our main contributions are as follows. To begin with, we build the relationship between the node deployment problem and minimum disk cover problem and prove node deployment problem to be NP-complete. Secondly, we, based on disk cover problem and Steiner tree problem, propose one new polynomial algorithm for node deployment, which aims at minimizing the number of all sensors for detecting and dealing with the events to reduce the cost.

The rest of the paper is organized as follows. In Section II, we introduce some notations and relevant problems. In Section III, we formulate node deployment problem with some constraint and prove it to be NP-complete, propose one strategy for node deployment for minimizing the number of sensors to reduce the cost. We also analyze the proposed polynomial algorithms in theoretical aspect. Simulations in Section IV validate the effectiveness of the proposed algorithm comparing with the strategies in [4]. In the end, we give some future work.

\section{Preiliminary}

In this paper, a graph $G=(V, E)$ is associated with a sensor network by associating a vertex of the graph with each sensor(the vertex set is $V$ ), and an edge of the graph with each sensor pair for which the inter-sensor distance is known(the edge set is $E$ ). We assume that $G$ is connected and has at least 4 vertices in the following parts.

Assuming that the monitoring field with side length $a$ meters is relatively large square area compared with the sensing range and communication range,. There are $n$ static sensors $V=\left\{v_{1}, v_{2}, \ldots, v_{\mathrm{n}}\right\}$ and $m$ mobile sensors $U=\left\{u_{1}, u_{2}, \ldots, u_{\mathrm{m}}\right\}$, which are deployed in the monitoring field. The network consisting of static sensors is called the stationary sensor network, and the network consisting of mobile sensors is called the mobile sensor network. The communication range of the static sensors and the mobile sensors are $r(r<<a)$ and $R(R \geq r)$, respectively, and we use the disc model for both sensing and communication.

Mobile sensors initially are deployed in a stationary manner and will move toward a static sensor or the sensing field of it after an event is detected by either some static sensors or mobile sensors. The moving speed of the mobile sensors equals $v(v<<a)$ meters per second and is much slower than the moving speed of mobile nodes. We introduce the following terms used in next section.

Steiner tree problem: Given a set $P$ of points in a metric space, the Steiner tree problem is to find the shortest network interconnecting the points in $P$; that is, the total length of edges in the network is minimal (the length of an edge is the distance between its two endpoints).

The Steiner tree problem is NP-hard [10], and the minimum spanning tree problem is polynomial-solvable (due to Kruskal [11] and Prim [12]).

Definition 1: Suppose an event happens at time $t_{1}$, it has been detected by the stationary sensor network at time $t_{2}$, and one mobile sensor receives the information at time $t_{3}$ (we can choose the earliest time for $t_{3}$ ), and finally the mobile sensor can reach the event region at time $t_{4}$. If the detection delay is defined as $T_{\text {detect }}=t_{2}-t_{1}$, the time of information transmission is $T_{\text {tra }}=t_{3}-t_{2}$ and the motion delay as $T_{\text {motion }}=t_{4}-t_{3}$, then the reaction delay is 
defined as $T=t_{4}-t_{1}$. The detection delay can be computed by the method in [5], so we will focus on the transmission time and the reaction time.

Note that since the radio transmission speed is much faster than the movement speed of the mobile sensors, for simplicity of analysis, the time spent on the information propagation part is sometimes omitted.

\section{Formulation and Deployment}

\subsection{Formulation}

We assume that the sensor field is made up of grid points, and the grid with side length $x$. The granularity is determined by the accuracy with which the sensor deployment is desired.

Let $P$ be a set of $n$ points in the plane. A disk $D$ covers a point $p \in P$ if the Euclidean distance between the center of $D$ and $p$ is no greater than the radius of $D$. The minimum disk cover problem is considered as follows: given a set $D$ of $n$ disks and a set $P$ of $m$ points in the Euclidean plane, where each disk covers a subset of points in $P$, to compute a subset of disks with minimum cardinality covering all the points in $P$. For short, we denote the minimum disk cover set problem by $\operatorname{MDC}$ and $\operatorname{MDC}(V, r)$ when $V$ denotes the vertex set, and $r$ denotes the radius of the disk.

The minimum disk cover problem is known to be NP-complete. The constant factor approximation algorithm which is due to Bronnimann and Goodrich (1995) [13] is the earlier work. Recently, a better algorithm is due to Liao and $\mathrm{Hu}$ [14] who study the planar minimum disk cover problem and propose an algorithm yielding a polynomial time approximation scheme. The new algorithm approximates the optimal disk cover set with a factor of $(1+\varepsilon)$ and run in $O\left(m n{ }^{O\left(\frac{1}{\varepsilon^{2}} \log ^{2} \frac{1}{\varepsilon}\right)}\right.$ ) time, where $\varepsilon>0$ can be as small as possible.

In addition to its theoretical interest, the minimum disk cover problem is applied in a mobile ad hoc network to avoid the notorious broadcast storm problem [15] and to save energy. We will apply the disk cover set problem as a useful method in the node deployment in hybrid wireless sensor networks.

Next, we will prove that the node deployment problem under constraint of coverage is NP-complete.

Theorem 1. The node deployment problem with coverage constraint is NP-complete.

Proof. Because we consider the node deployment problem with coverage constraint, which means the sensors need cover the monitoring field for the events which can happen anywhere in the monitoring field. Comparing with the MDC, we can observe the node deployment problem containing MDC. Because the node deployment problem means to cover every point in the monitoring field, and the MDC means cover some given points. The minimum disk cover problem is NP-complete, therefore, the node deployment problem also is.

This finishes the proof.

\subsection{Node Deployment Algorithm}

Firstly, we can divide the monitoring field into grids, and, in order to cover the monitoring field, we can use one grid center to represent each grid, so the node deployment problem can be converted into MDC. However, we can just cover the monitoring field with high probability. Next, we need consider how to enhance the connectivity of the wireless sensor networks by adding minimal mobile sensors.

The second step of our algorithm aims at constructing a connected wireless sensor network by adding minimal mobile sensors. We assume the transmission range of the mobile sensors is $R$, and $R \geq r$.

Now, we need consider a variation of the Steiner tree problem for minimal Steiner points, and this problem has many applications in wireless communication [16,17].The problem is more formally as follows. 
Problem 1. Steiner Tree Problem for Minimal Steiner Points (STP-MSP) [18]:

Instance A set $\mathrm{P}=\left\{t_{1}, t_{2}, \ldots, t_{\mathrm{n}}\right\}$ of terminals in a Euclidean plane $\boldsymbol{R}^{2}$ and a constant $r>0$.

Solution A Steiner tree $T$ interconnecting all terminal points such that each edge in $T$ has length no more than

$r$.

Objective Minimizing the number of Steiner points in $T$.

Therorem 2 [19]. The Steiner tree problem for minimal Steiner points is NP-complete.

The Minimum Spanning Tree (MST) can be developed as a approximation algorithm for Steiner tree problem for minimal Steiner points as follows [18].

Algorithm 1. Steinerized Minimum Spanning Tree Algorithm

Step1 Construct an MST $T_{\mathrm{mst}}$ of $\mathrm{P}$ and set $T:=T_{\mathrm{mst}}$.

Step2 for all e in $T$ whose length $l(e)>r$ do begin

Cut long edge e into $\lceil l(e) / r\rceil$ shorter ones of equal length by placing $(\lceil l(e) / r\rceil-1)$ Steiner points on e.

Replace edge e with $\lceil l(e) / r\rceil$ shorter edges.

end for

Output Steiner tree $T_{\text {smst }}:=T$.

Therorem 3 [19]. The Steinerized minimum spanning tree is a polynomial-time approximation with performance ratio exactly 4 .

For convenience, let $u$ and $v$ be two points in the plane, and $[u, v]$ denotes the line segment connecting $u$ and $v$, and $d(u, v)$ denotes the Euclidean distance between $u$ and $v$. Two static sensor nodes $u$ and $v$ can communicate with each other if $d(u, v) \leq r$, two mobile sensor nodes $u$ and $v$ can communicate with each other if $d(u, v) \leq R$. A static sensor node $u$ can communicate with a mobile sensor node $v$ if $d(u, v) \leq r$.

Based on MDC and STP-MSP, our algorithm as follows.

Algorithm 2. Node Deployment Algorithm

INPUT: A square field in the Euclidean plane with side length $a$, and positive real constants $r>0$ and $R \geq r$ representing the communication ranges of static sensors and mobile sensors, respectively, an approximation algorithm for MDC and an approximation algorithm for MST.

OUTPUT: $P$ and $Y$

Step1 Divide the monitoring field into grids, and use one grid center to represent each grid. Let $V=\left\{x_{1}, x_{2}, \ldots, x_{n}\right\}$ be the set of the centers of the grids in Euclidean plane $\mathrm{R}^{2}$.

Step2 Apply the approximation algorithm for MDC to $V$ to obtain a point set $P=\left\{t_{1}, t_{2}, \ldots, t_{m}\right\}$ that is a feasible solution to $\operatorname{MDC}(V, r)$. Without loss of generality, we assume that $\mathrm{P}$ is minimal, meaning that no proper subset of $P$ is a feasible solution for $\operatorname{MDC}(V, r)$.

Step3 Let $G=(V, E, w)$ be an undirected edge-weighted complete graph on the vertices in $V$, where for $1 \leq i<j \leq n$, the weight of edge $e=x_{i} x_{j}$ is $w\left(x_{i} x_{j}\right)=\mathrm{d}\left(x_{i}, x_{j}\right)$, where $d\left(x_{i}, x_{j}\right)$ is the distance between $x_{i}$ and $x_{j}$ in the Euclidean plane.

Step4 Construct an MST $T_{\mathrm{mst}}$ of P and set $T:=T_{\mathrm{mst}}, k:=0$.

Step5 for each edge $e=x_{i} x_{j}$ in $T$ with $\mathrm{d}\left(x_{i}, x_{j}\right)>r$ do begin

if $r<\mathrm{d}\left(x_{i}, x_{j}\right) \leq 2 r$ then

$k:=k+1$. Place a Steiner point $y_{k}$ at the midpoint of the line segment $\left[x_{i}, x_{j}\right]$, 
else if $\quad d(x i, x j)>2 r \quad$ then

Place two Steiner points on the line segment $[\mathrm{xi}, \mathrm{xj}]: \mathrm{yk}+1$ with distance $r$ to $x i$, and $y k+2$ with distance $r$ to $\mathrm{xj}$.

Cut long edge $e=y k+1 y k+2$ into shorter ones of equal length by placing $(\lceil(d(x i, x j)-2 r]) / R\rceil-1)$ Steiner points $y k+3, y k+4, \ldots, y k+[(d(x i, x j)-2 r]) / R\rceil+1$ on the line segment $[y k+1, y k+2]$, cutting the line segment into $\lceil(\mathrm{d}(\mathrm{xi}, \mathrm{xj})-2 \mathrm{r}) / \mathrm{R}\rceil$ equal parts.

$k:=k+\left\lceil\left(d\left(x_{i}, x_{j}\right)-2 r\right) / R\right\rceil+1$

end if

end for

Step 6 Output $P=\left\{t_{1}, t_{2}, \ldots, t_{\mathrm{m}}\right\}$ and $\mathrm{Y}=\left\{y_{1}, y_{2}, \ldots, y_{\mathrm{k}}\right\}$

The position of each static node in hybrid sensor network corresponds to the location of the points in set $P$, and the elements in set $Y$ exactly are corresponding to the mobile sensors.

Theorem 4. The hybrid wireless sensor network produced by Algorithm 2 is connected and can reach full-coverage when the side length $x$ of each grid varies to 0 , which implies that the network can reach full-coverage with high probability when $x$ is small.

Proof. First, we prove the connectivity of the network.

We construct the topology graph of the sensors deployed in the network. Let $G=(V, E)$ be the associate communication graph of the network.

By Step 4 and Step 5, we can easily construct a spanning tree $T$ of G. Because we obtain a tree of the Euclidean graph by Step 4 and Step 5, if we can prove that the edge length is no more than the transmission range required, we can get a spanning tree of the communication graph which has the same topology with the one in Euclidean plane. In Step 5, we add nodes such that the distance between consecutive nodes does not exceed the corresponding transmission range, so the network is connected.

Second, we prove the coverage of the network.

When the side length $x$ of the grid varies to 0 , that means, the grid becomes one point; so for any point in the monitoring field, there exists one disk to cover it. Hence, the network can reach full-coverage with high probability when $x$ is small.

This finishes the proof.

It is noted that the approximation algorithm for MDC in [16] proposed by Liao and Hu is a polynomial time approximation scheme, and for any given constant $\varepsilon>0$, it can produce an $(1+\varepsilon)$-approximation of the optimal solution and run in $O\left(m n O\left(\frac{1}{\varepsilon^{2}} \log ^{2} \frac{1}{\varepsilon}\right)\right.$ time.

Assumptions: We apply the algorithm for MDC proposed by Liao and $\mathrm{Hu}$, and the algorithm for MST proposed by Kruskal in Algorithm 2.

Theorem 5. The time complexity of the Algorithm 2 is polynomial for node deployment in hybrid wireless sensor networks.

Proof: By our assumptions above, the Step 2 and Step 4 are both polynomial time. Step 5 is similar to Algorithm 1, which is polynomial time by Theorem 2, so we can get the time complexity of algorithm 2 is polynomial.

This finishes the proof.

\section{Simulations and Evaluation}

In this section, we conducted extensive simulations to study the performance of our sensor deployment scheme. 


\subsection{Methodology and Parameters}

Assuming that a fixed square area of sensor network with side length $a$. The transmission range of static sensor nodes and mobile nodes, are set to be $r=5 \mathrm{~m}$ and $R=8 \mathrm{~m}$ as a default value, respectively. Our simulation is based on TIM, and we define the required reaction delay to be $r / v$, where $r$ denotes the transmission range of the static sensors and $v$ denotes the default moving speed of the mobile sensors, which is set to be $0.5 \mathrm{~m} / \mathrm{s}$ based on general knowledge.

\subsection{Number of Sensor Nodes Deployed in the Moniter Field}

Here the side length of the monitor area, $a$, is set to be $100 \mathrm{~m}$. We collect the number of sensor nodes conducted based on our algorithm as the side length of each grid increases, ranging from $2 \mathrm{~m}$ to $10 \mathrm{~m}$. We evaluate the efficiency of our hybrid sensor deployment algorithms.

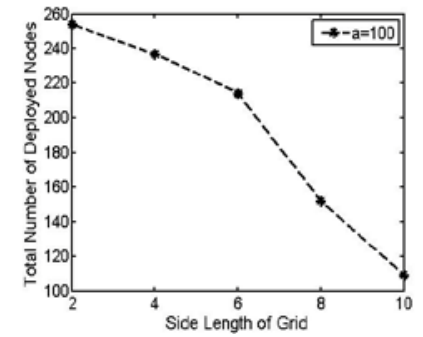

Fig. 1

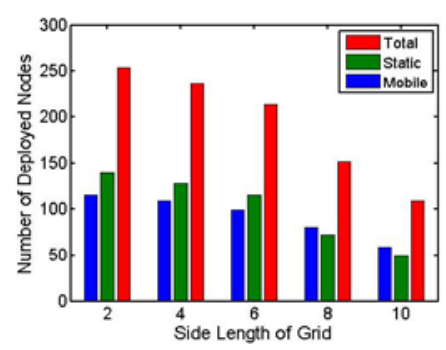

Fig. 2.

As illustrated in Fig. 1, the total number of sensor nodes conducted by our algorithm decreases when the side length of each grid increases. Moreover, the number of mobile sensors becomes relatively larger than that of static sensors as showed in Fig. 2. Obviously, as the side length of grid increases, the mobile sensors supporting the connection between static sensors play an important role in the perspective of quantity.

\subsection{Reaction Delay by Hybrid Wireless Sensor Network}

Finally, we evaluate the reaction delay of our algorithm. Here we focus on the motion delay since the detection delay and the transmission delay can be omitted in our assumption that they are relatively small compared with motion delay with an optimal node scheduling method. 


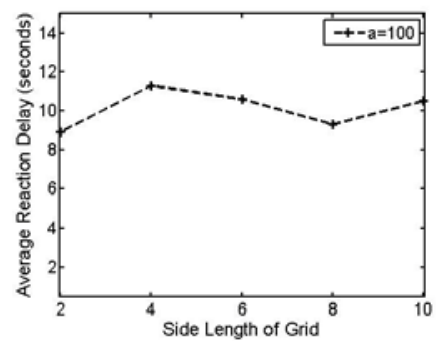

Fig. 3.

We randomly conducted a hundred of target events in the monitor region, and get the average reaction delay within twelve seconds, see Fig. 3..

\section{Conclusion and Future work}

Node deployment in mission-oriented WSNs is becoming more important, and we give one polynomial algorithm for node deployment considering the cost saving, the approximation algorithms for node deployment are needed when given the number of the mobile sensors. Therefore, we will consider how to deploy the sensors to achieve the minimum relay time while the number of the mobile sensors is fixed in the future work.

\section{Acknowledgment (Heading 5)}

This work is supported in part by the China NSF grants (60825205, 61003224), China 973 project (2006CB303000), Natural Science Foundation of Hohai University (2008428511), China Postdoctoral Science Foundation (20090461087), and the Fundamental Research Funds for the Central Universities (2009B21414).

\section{References}

[1] S. S. Dhillon, K. Chakrabarty, and S. S. Iyengar, "Sensor placement for grid coverage under imprecise detections," Proc. of the $15^{\text {th }}$ Intel. Conference on Information Fusion, vol. 2, pp. 1581-1587, July 2002.

[2] S. S. Dhillon and K. Chakrabarty, "Sensor placement for effective coverage and surveillance in distributed sensor networks,” Proc. of IEEE WCNC, vol. 3, pp. 1609-1614, 2003.

[3] K. Chakrabarty, S. S. Iyengar, and H. Qi, "Grid coverage for surveillance and target location in distributed sensor networks,” IEEE Trans. Computers, vol. 51, pp. 1448-1453.

[4] X. Bai, D. Xuan, Z. Yun, T.H. Lai, and W. Jia, "Complete optimal deployment patterns for full-coverage and $k$-connectivity $(k \leq 6)$ wireless sensor networks”, In ACM Mobihoc, pp. 401-410, 2008.

[5] T.-L. Chin, P. Pamanathan, and K. K. Saluja, "Analytic modeling of detection latency in mobile sensor networks,” In ACM IPSN, 2006.

[6] B. Liu, P. Brass, O. Dousse, P. Nain, and D. Towsley, “Mobility improve coverage of sensor networks,” In ACM MobiHoc, 2005.

[7] N. Bisnik, A. Abouzeid, and V. Isler, "Stochastic event capture using mobile sensors subject to a quality metric,” In ACM MobiCom, 2006.

[8] G. Xing, J. Wang, K. Shen, Q. Huang, X. Jia and H. C. So, "Mobility-assisted spatiotemporal detection in wireless sensor networks,” In IEEE ICDCS, 2008. 
[9] S. Gandhi, S. Suri, and E. Welzl, "Catching elephants with mice: Sparse sampling for monitoring sensor networks,” In ACM SenSys, 2007.

[10] M. R. Garey, R. L. Graham, and D. S. Johnson, "The complexity of computing Steiner minimal trees," SIAM J. Applied Math., 32(4): 835-859, 1977.

[11] J. B. Kruskal, “ On the shortest spanning subtree of a graph and the traveling salesman problem,” In Proc. of American Mathmatics Society, 7(1): 48-50, 1956.

[12]R. C. Prim, "Shortest connection networks and some generalization," Bell System Technical Journal, 36: 1389-1401, 1957.

[13]H. Brönnimann and M. T. Goodrich, “Almost optimal set covers in finite VC-dimension,” In Proc. of the 10th Computational Geometry, pp. 293-302, 1994.

[14] C. Liao and S. Hu, "Polynomial time approximation schemes for minimum disk cover problems,” J. Comb. Optim, published online, February 28, 2009.

[15] G. Calinescu, I. I. Mandoiu, P.-J. Wan, and A. Z. Zelikovsky, "Selecting forwarding neighbors in wireless ad hoc networks,” ACM Mob. Netw. Appl. 9: 101-111, 2004.

[16]C.-S. Li, F. F. Tong, C. J. Georgiou, and M. Chen, "Grain equalization in metropolitan and wide area optical networks using optical amplifiers,” In Proc. IEEE INFOCOM, pp. 130-137, 1994.

[17] B. Bamamurthy, J. Iness, and B. Mukherjee, " Minimizing the number optical amplifer needed to support a multi-wavelength optical LAN/MAN,” In Proc. IEEE INFOCOM, pp. 261-268, 1997.

[18]G. Lin and G. Xue, "Steiner tree problem with minimum number of Steiner points and bounded edge-length,” Information Processing Letters, 69: 53-57, 1999.

[19]D. Chen, D. Z. Du, X. D. Hu, L. Wang, and G. Xue, "Approximations for Steiner trees with minimum number of Steiner points,” Journal of Global Optimization, 18: 17-33, 2000. 